TPI-MINN-99/58-T

UMN-TH-1830/99

\title{
Calculating the tension of domain wall junctions and vortices in generalized Wess-Zumino models.
}

\author{
M. Shifman and T. ter Veldhuis \\ Theoretical Physics Institute, Univ. of Minnesota, Minneapolis, MN 55455
}

\begin{abstract}
We study BPS saturated objects with axial geometry (wall junctions, vortices) in generalized Wess-Zumino models. It is observed that the tension of such objects is negative in general (although "exceptional" models are possible). We show how an ambiguity in the definition of central charges does not affect physical quantities, and we comment on the stability of the junctions and vortices. We illustrate these issues in two classes of models with $Z_{N}$ symmetry. On the basis of analytical large $N$ calculations and numerical calculations at finite $N$, we argue that the domain wall junctions in these models are indeed BPS saturated, and we calculate the junction tensions explicitly.
\end{abstract}

E-mail: shifman@physics.spa.umn.edu, veldhuis@hep.umn.edu 


\section{Introduction}

Studying the BPS domain walls in supersymmetric theories is interesting, especially in gauge theories [1], because one can get information on non-trivial dynamical features. For example, it was found that the tension of such domain walls determines the decay rate of "false "vacua in large $N$ supersymmetric gluodynamics [2, 3].

It was noted [4] that theories with either a $U(1)$ or $Z_{N}$ global symmetry may contain BPS objects with axial geometry. Vortex-like objects which conserve 1/4 of the original supersymmetry were considered in [5], while domain wall junctions of the hub and spoke type, which also preserve $1 / 4$ of supersymmetry, were discussed in [4, 6, [7]. General considerations of the tensorial central charges responsible for the saturation of the vortices and junctions were presented in [8, 9, 10]. The existence of BPS wall junctions also leads to non-trivial dynamical consequences. For instance, in [11] the large $N$ behavior of the BPS junction tension was used to argue in favor of soliton-like heavy hadrons with mass proportional to $N$ in supersymmetric gluodynamics.

The study of possible dynamical implementation of the BPS wall junctions has just begun. Apart from the original works in which the existence of such junctions was first noted, they were discussed in 111, 12, 13, 14]. In [11] a generalized WessZumino model, which we will refer to as Model I, with one chiral superfield $X$ and the superpotential

$$
\mathcal{W}=N\left\{X+\frac{N}{N+1}\left(\frac{X}{N}\right)^{N+1}\right\},
$$

was considered. This model is related to the Veneziano-Yankielowicz [15] effective Lagrangian, which, in turn, models supersymmetric Yang-Mills theory. The parameter $N$ is an integer that corresponds to the the number of colors of the gauge group $\mathrm{SU}(N)$ of SUSY gluodynamics. The superpotential (1) was originally suggested for that purpose in [16]. At any given $N$ it possesses a $Z_{N}$ symmetry which is spontaneously broken. In the large $N$ limit an analytic solution for the BPS domain walls was found in [17. The most essential feature of the solution is the fact that the wall width scales as $N^{-1}$, and vanishes at $N \rightarrow \infty$. This result, in conjunction with two BPS junction configurations presented in [11] at $N=\infty$, implies that BPS junctions do exist at large $N$ in the theory with superpotential (1). The junction tension was found to scale as $N^{2}$ at large $N$.

In [13] the first analytic solution for a BPS junction was found in a specific generalized Wess-Zumino model. Among stimulating findings in this work is the fact that the junction tension turned out to be negative in this model. The model has $Z_{3}$ symmetry. It is derived from a $\mathrm{SU}(2)$ Yang-Mills theory with extended supersymmetry $(\mathcal{N}=2)$ and one matter flavor perturbed by an adjoint scalar mass. The original model contains three pairs of chiral superfields and, in addition, one extra chiral superfield. In fact, for the purpose of studying the BPS walls and wall junctions, the model can be further simplified. It can be easily stripped of its inessential features and cast into the form of a $Z_{N}$ symmetric model. ( $N$ is an 
arbitrary integer, not necessarily equal to 3$)$. The generalized and simplified model, which we refer to as Model II, contains $N$ chiral superfields $M_{k}$ with $k=1,2, \ldots, N$ and another chiral superfield $T$. The superpotential is

$$
\mathcal{W}=N\left\{-A\left[\sum_{k=1}^{N}\left(T-N q^{k-1} e^{-\pi i / N}\right) M_{k}^{2}\right]+B T\right\},
$$

where $q=e^{i 2 \pi / N}$. The constants $A$ and $B$ are assumed to be $N$ independent and positive.

Model I is invariant under the transformation

$$
X(x, \theta) \rightarrow e^{2 \pi i k / N} X\left(x, \theta^{\prime}\right), \quad \theta^{\prime}=e^{-\pi i k / N} \theta
$$

the corresponding transformation of the superpotential is

$$
\mathcal{W}(X(x, \theta)) \rightarrow e^{2 \pi i k / N} \mathcal{W}\left(X\left(x, \theta^{\prime}\right)\right)
$$

Model II is invariant under the transformation

$$
T(x, \theta) \rightarrow e^{2 \pi i k / N} T\left(x, \theta^{\prime}\right), \quad M_{l}(x, \theta) \rightarrow M_{l-k}\left(x, \theta^{\prime}\right) \quad \theta^{\prime}=e^{-\pi i k / N} \theta ;
$$

the corresponding transformation of the superpotential is

$$
\mathcal{W}(T(x, \theta), M(x, \theta)) \rightarrow e^{2 \pi i k / N} \mathcal{W}\left(X\left(x, \theta^{\prime}\right), M\left(x, \theta^{\prime}\right)\right) .
$$

Model I is among the simplest supersymmetric models allowing one to study BPS junctions and calculate tensions. T The model has $N$ distinct vacua in which the scalar component of $X$ takes the vacuum expectation value $N e^{i \pi(2 k-3) / N}$, with $k=1,2, . ., N$. The perturbative spectrum consists of a chiral multiplet with mass $m=N$.

Let us now briefly discuss the vacuum structure and spectrum of Model II. In this model there are $2 N$ distinct, physically equivalent vacua. In these vacua the scalar components of the $T$ and $M$ fields take the vacuum expectation values

$$
T=N e^{i \pi(2 k-3) / N}, \quad M_{k}= \pm \sqrt{\frac{B}{A}}, \quad M_{l}=0 \quad \text { for } \quad l \neq k
$$

with $k=1,2, . ., N$. The perturbative spectrum contains two chiral multiplets, linear combinations of the field $T$ and the field $M_{k}$ that obtains a vacuum expectation value, with mass $m_{1,2}=2 \sqrt{A B} N$, and $(N-1)$ chiral multiplets with mass

$$
m_{k}=4 N^{2} A(1-\cos \pi(k-1) / N)
$$

for $k=2,3, . ., N$.

\footnotetext{
${ }^{1}$ It is worth noticing that in a different context its 2-dimensional reduction was studied in [18], where it was shown to be integrable.
} 
Apart from the $Z_{N}$ symmetry, Eq.(5), Model II has additional $Z_{2}^{N}$ symmetry, since any of the fields $M_{k}$ can be transformed as $M_{k} \rightarrow-M_{k}$. The superpotential in Eq.(2) is obviously invariant under this transformation. In our consideration of the wall junctions below we will disregard the $Z_{2}^{N}$ symmetry, limiting ourselves just to a $Z_{N}$ family of vacua. (This corresponds to the choice of the + sign in front of $\sqrt{A / B}$ in Eq.(7)). The walls and junctions of the type where (some of) the vacua lie outside this family may or may not be BPS saturated. We leave this issue open for future investigations.

Needless to say that in both models I and II the walls connecting the distinct vacua and the corresponding junctions are topologically stable. (The issue of stability of the BPS junctions is discussed separately in Sect.(77).) They need not be BPS saturated, generally speaking. We know, empirically, that sometimes in certain models equations of the BPS saturation have no solutions with the proper boundary conditions 118, 19] even in the case of isolated walls, let alone the wall junctions.

It was known previously that the isolated walls in Model I are saturated, see [16. In Appendix A we give an analytical solution to the BPS equation for the domain walls in Model II for a specific ratio of the parameters $A$ and $B$; we have verified numerically that these walls also saturate the BPS bounds for other values of the parameters. In Sect.(6) we provide evidence that the domain wall junctions in Models I and II are also BPS saturated. Were the walls and junctions nonsaturated, one could still calculate the relevant central charges. They would then represent lower bounds on the corresponding tensions.

Most considerations in this paper concern the basic domain wall junction, the junction with $N$ sectors in a model with $Z_{N}$ symmetry. But in Appendix B we present an analytic solution to the BPS equation for the class of triple junctions in Model II for a specific ratio of the parameters $A$ and $B$, and $N$ a multiple of three. For $N=3$, the basic junction and the triple junction coincide. Non-basic junctions in Model I are studied in [14. Networks of domain walls in Model I are studied in 20, 21].

In this paper we consider BPS wall junctions and calculate their tensions in Models I and II specified above. We also consider general aspects, including the sign of the tension, the ambiguity of the central charges, and stability, that are valid beyond Model I and II. These models are used to illustrate the general assertions.

The organization of our paper is as follows. In Sect.(2) we describe a general method to calculate the BPS bound on the tension of domain wall junctions. We show that the junction tension is typically negative, although we do not exclude exotic models with positive tension. We then use this method to explicitly calculate the junction tension in Models I and II. (Secs. 3 and 4). For Model I we calculate the junction tension analytically in next to leading order in $1 / N$, and numerically for finite values of $N$. For Model II the junction tension is calculated analytically for all values of $N$ and all values of the parameters $A$ and $B$. In Sect.(5) we show that ambiguities in the central charges cancel in the calculation of the junction tension, and we illustrate this cancellation by recalculating the tension in Model II with a 
different definition of the central charges. In Sect.(6) we demonstrate by numerical - and in certain limits, analytical - calculations that in both models I and II the wall junctions are, in fact, saturated. In Sect.(7) we comment on the stability of the

BPS junctions. The appendices contain analytical results for domain walls and the triple junction in Model II.

\section{Generalities of the solutions with the axial ge- ometry}

For both Models I and II it is possible to calculate, using the general theory of the central charges, the BPS bounds on the junction tension. The general formula worked out in [10] implies

$$
\frac{M}{L}=-\oint a_{k} d x_{k}+2 \oint d n_{k} S_{k},
$$

where $a_{k}$ is an axial current. At the classical level only the component of the current built from the scalar fields is important. Moreover,

$$
S_{1,2}=\{\operatorname{Re} \Sigma, \operatorname{Im} \Sigma\}
$$

where $\Sigma$ is related to the superpotential. The integrals run over the large circle (see Fig.(西)) with $R \rightarrow \infty$, and $d \vec{x}(d \vec{n})$ is an infinitessimal vector along (perpendicular to) the contour. The precise definitions of $a_{k}$ and $\Sigma$ have (correlated) ambiguities. The sum in Eq.(9) is unambiguous, however [10]. The above ambiguity can be exploited to optimize the calculation of the junction tension. This will be explained below in two particular applications, Model I and II.

We will consider the wall junction of the "hub and spoke" type. The following conventions are convenient (although not necessary). We will orient the wall spokes as indicated in Fig.(1), namely, the hub is at the origin, the "first" spoke runs along the $\hat{x}$ axis in the positive direction, the "second" runs at an angle $2 \pi / N$, and so on. In the point $\mathrm{P}$ the theory "sits" in the first vacuum, in the point $\mathrm{Q}$ in the second, etc. This configuration is topologically stable.

We will assume that the $Z_{N}$ symmetry is realized through multiplication of (some of) the fields by a phase. This is certainly true in Models I and II. If on the junction solution (the lowest components of) the fields $X, T$ (generically, $\Phi$ ) rotate as

$$
\Phi\left(\zeta e^{2 \pi i k / N}\right)=e^{2 \pi i k r / N} \Phi(\zeta)
$$

we will say that the rotation weight of the field $\Phi$ is equal to $r$. Configurations with the positive rotation weight will be referred to as junctions (vortices), while those with the negative weight will be referred to as anti-junctions (anti-vortices). It is worth reminding that

$$
\zeta=x+i y, \quad \partial_{\zeta}=\frac{1}{2}\left(\partial_{x}-i \partial_{y}\right)
$$




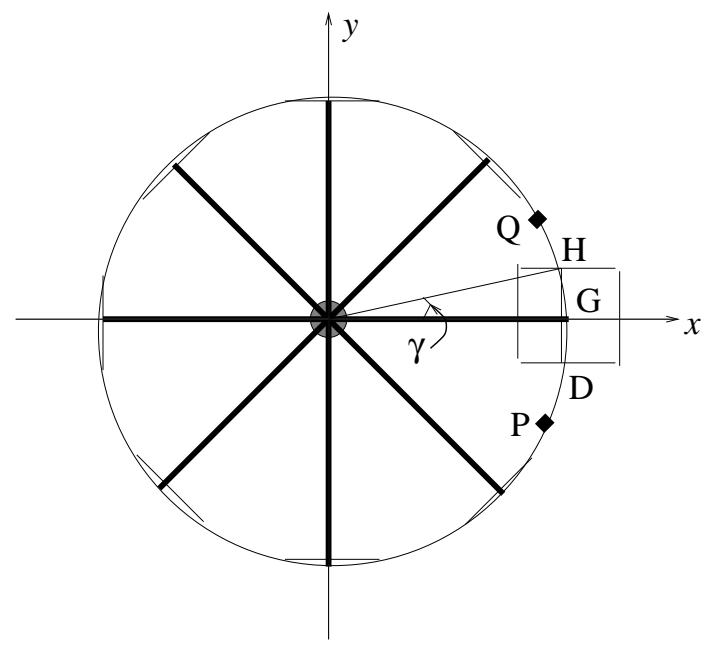

Figure 1: A "standard" convention regarding the wall junction. The large circle of radius $R$ enters the definition of $E(R)$ while the circle with the segments removed is relevant to $\tilde{E}(R)$. The box of size $L \times L$ around the point $\mathrm{G}$ is blown up in Fig.(2). $L$ is an auxiliary parameter chosen to satisfy the constraint $l \ll L \ll R$.

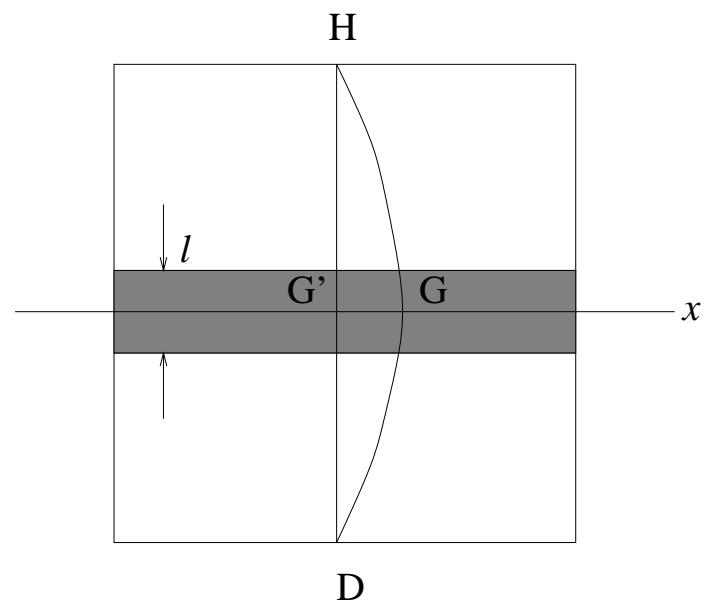

Figure 2: A blow up of the box in Fig.(1). The shaded rectangle represents the wall, the length of the interval GG' is of order $L^{2} / R$. 
The BPS wall junctions satisfy the equations

$$
2 \partial_{\zeta} \Phi_{k}=e^{i \delta} \frac{\partial \overline{\mathcal{W}}}{\partial \bar{\Phi}_{k}}
$$

where the phase $\delta$ depends on the phase of the superpotential (see below). The equations for anti-junctions is

$$
2 \partial_{\bar{\zeta}} \Phi_{k}=e^{i \tilde{\delta}} \frac{\partial \overline{\mathcal{W}}}{\partial \bar{\Phi}_{k}}
$$

i.e. $\zeta$ is replaced by $\bar{\zeta}$. Thus, in our convention the phase of $\Phi$ grows in the anticlockwise directions for the junction, and in the clock-wise direction for the anti-junction.

The superpotential can always be chosen in such a way that in the vacuum $\mathrm{P}$ its phase is $\exp (-i \pi / N)$ while in the vacuum $\mathrm{Q}$ it is $\exp (i \pi / N)$. See Fig.(11) for the definition of $\mathrm{P}$ and $\mathrm{Q}$. (Note that $\operatorname{Re}\{\mathcal{W}\}$ is then positive in both vacua). Then the phase $\delta$ in Eq. (13) must be set to zero in both Models I and II.

For the wall junctions the rotation weights of $X$ and $T$ are obviously equal to 1 .

Now, we proceed to discuss general features of the tension associated with wall junctions. In Fig.(1) the energy of the junction configuration (per unit length) is defined as the integral over the volume energy density over the area inside the circle, where it is assumed that the circle radius $R$ tends to infinity,

$$
E(R)=\frac{M}{\text { length }}=\int_{|\vec{r}| \leq R} \mathcal{H}(x, y) d x d y=T_{1} R+T_{2}+\ldots, \quad R \rightarrow \infty .
$$

Here the dots denote terms vanishing in the limit $R \rightarrow \infty$. It is tempting to say that $T_{2}$ is associated with the $(1 / 2,1 / 2)$ central charge while $T_{1}$ with the $(1,0)$ central charge (see Eq.(9), the first and second term, respectively). We hasten to note that these central charges, being considered individually, are ambiguous [10]. It is only the combination in Eq.(9) which is fixed unambiguously. It is intuitively clear that $T_{1}=N T_{\text {wall }}$ where $T_{\text {wall }}$ is the tension of the isolated wall. Moreover, it is also clear that $T_{2}$ (which will be also referred to as $T_{\text {junction }}$ ) is typically negative. Indeed, from Eq.(15) it follows that, for small $R, E(R)=c R^{2}$, where $c$ is a numerical coefficient. At large $R$ the quadratic dependence on $R$ changes to linear, see Eq.(15). Matching these two scaling laws straightforwardly, connecting the parabola and the straight line at the value of $\mathrm{R}$ where their slope is equal, we conclude that $T_{2}$ is forced to be negative. Models where it could be positive would require, in essence, two scales and an intermediate regime between the parabolic and linear regime. In order to illustrate the typical situation, we plot the energy $E$ as a function of $R$ for Model II with $N=3, B=\sqrt{3}$ and $A=B / 9$ in Fig.(3).

Actually, this figure gives the energy $E$ defined as the integral over $\mathcal{H}(x, y)$ over the equilateral triangle with distance $R$ from the center to each of the sides instead of the energy in a circle. The orientation of the triangle is such that each of the sides is perpendicular to a spoke. We made this modification because for this triangle 


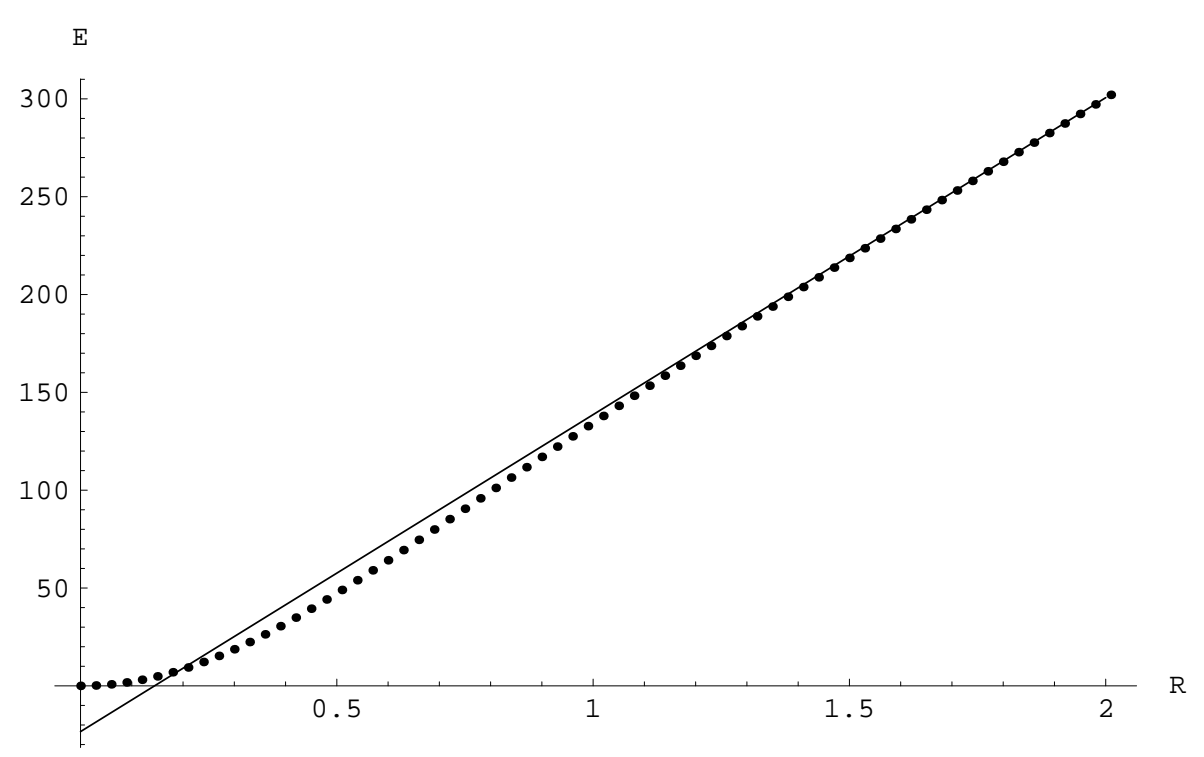

Figure 3: Energy $E$ as a function of radius $R$ (dotted line) for the triple junction of model II with $N=3, A=B / N^{2}$ and $B=\sqrt{3}$, compared to the line $E=T_{1} R+T_{2}$ (solid line). This example illustrates the typical junction with negative tension.

the energy converges to the linear relation Eq.(15) exponentially fast, whereas for a circle the departure from the linear behavior is of the order $1 / R$. (As explained below, the coefficient of the $1 / R$ term is just a geometrical factor taking into account the curvature of the circle as it intersects the domain wall.)

For the particular values of the parameters that we chose, there is an analytical wall junction solution of the BPS equations, which is presented in Appendix B. The energy $E(R)$ can therefore be calculated exactly and is shown in Fig.(3). It is clear from Fig.(3) that even though there is a change from a convex to a concave regime, $E(R)$ converges to the linear regime so fast that $T_{\text {junction }}$ is negative. The tensions can be calculated analytically and take the values $T_{1}=162$ (see Sect.(6)) and $T_{2}=-27 \sqrt{3} / 2$ (see Sect.(国)). Similar graphs for Model I junctions are presented in Ref.[14]. For comparison, we sketch what a similar graph would look like for an exotic model with positive junction tension in Fig.(4).

Below we give a general prove of the fact that $T_{1}=N T_{\text {wall }}$, and illustrate more quantitatively that $T_{\text {junction }}$ is negative in both Models I and II, and in other "natural" models. Of crucial importance is the fact that the wall width (i.e. the transverse dimension inside which the energy density is nonvanishing, while outside it vanishes with exponential accuracy) is finite (i.e. $R$ independent). This width is denoted by $l$, see Figs. (11) and (2). In both Models I and II, $l$ is actually $O(1 / N)$. At the moment we do not assume $N$ to be large. It can be any integer $>2$. If $R \rightarrow \infty$, one can replace the integration domain in Eq. (15) - the interior of the circle can be replaced by the interior of the circle with the segments removed. If $\tilde{E}(R)$ is defined as the integral over the interior of the circle with the segments removed, the difference 


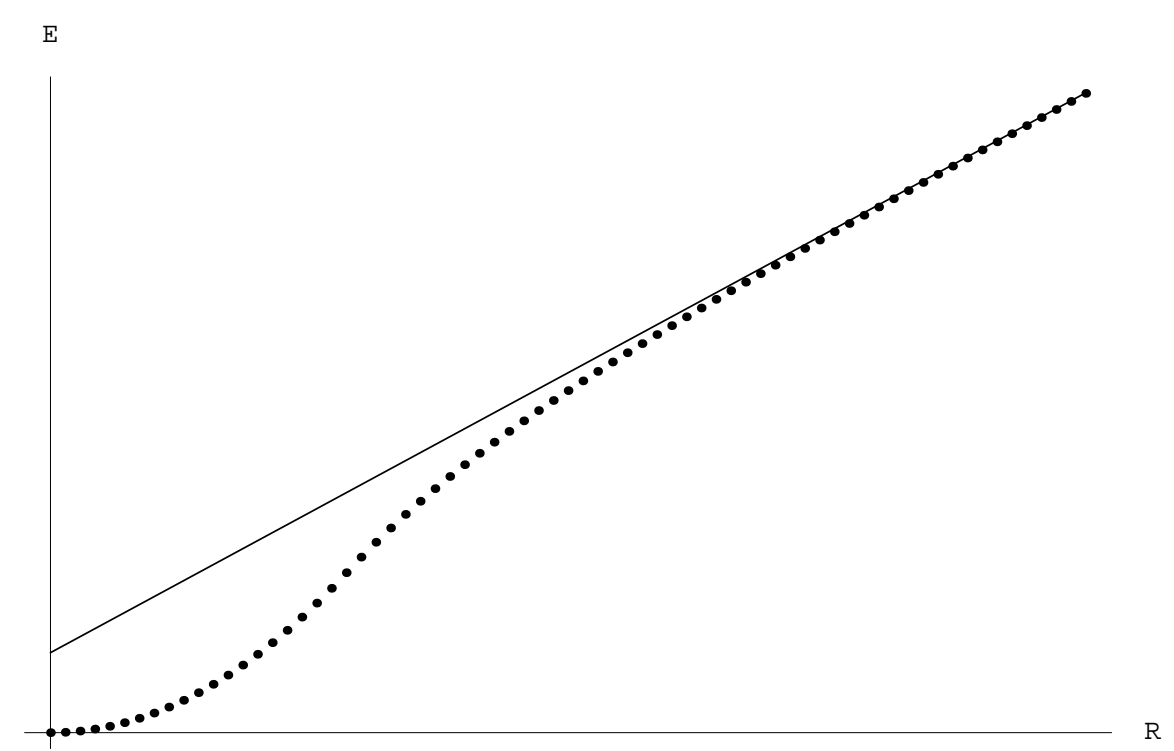

Figure 4: Sketch of the energy $E$ as a function of radius $R$ for a hypothetical, exotic model with the positive junction tension.

$\tilde{E}(R)-E(R)$ tends to zero in the limit $R \rightarrow \infty$. (The length of the straight line $\mathrm{DH}$ is $L$, where $L$ is an arbitrary parameter, $l \ll L \ll R$.) This can be clearly seen from Fig.(2), which presents a detail of the domain near the point G. The area of the removed segment is $O\left(L^{3} / R\right)$. The parameter $L$ does not scale with $R$ when $R$ tends to $\infty$, hence the area of the removed segment tends to zero. Since the volume energy density is finite, the difference $\tilde{E}(R)-E(R) \sim R^{-1}$.

For the BPS saturated configuration the energy $E(R)$ or $\tilde{E}(R)$ can be rewritten as a combination of the corresponding contour integrals. The master equation for the wall junction is presented in Eq.(9). It is assumed in this equation that the phase $\delta$ in Eq. 19 is zero, which is the case with our convention. There is an ambiguity in $\Sigma$ and $a_{k}$ in this master formula: a certain reshuffling is possible due to the ambiguity in the definition of the supercurrent and the energy-momentum tensor. This is explained in great detail in Ref. [10]. We will return to the discussion of this ambiguity later on. For the time being let us stick to the canonic definitions. Then

$$
\Sigma=\mathcal{W}, \quad S_{1,2}=\{\operatorname{Re} \mathcal{W}, \operatorname{Im} \mathcal{W}\}
$$

and

$$
a_{k}=\frac{1}{2} \sum_{\ell}\left(\Phi_{\ell} i \partial_{k} \bar{\Phi}_{\ell}-\bar{\Phi}_{\ell} i \partial_{k} \Phi_{\ell}\right) \equiv \frac{1}{2} \sum_{\ell}\left(\Phi_{\ell} i \stackrel{\leftrightarrow}{\partial_{k}} \bar{\Phi}_{\ell}\right)
$$

where $k=1,2$. The sum runs over all scalar fields involved in the solution. Fields which are purely real obviously drop out. Only complex fields contribute; for such fields, if $\Phi=\rho e^{i \eta}$,

$$
a_{k}=\rho^{2} \partial_{k} \eta .
$$


Note that Eq. (17) for the current appearing in the master equation is valid for the wall junctions. For the anti-junctions one must reverse the sign in Eqs. (17) and (18).

How do we see that with our convention the phase $\delta$ in Eq. (13) is zero? To this end, let us consider this equation far away from the "hub", on the line DH. On this line Eq. (13) becomes

$$
\partial_{y} \Phi=i \frac{\partial \overline{\mathcal{W}}}{\partial \bar{\Phi}} .
$$

Since $\Delta \mathcal{W}=\mathcal{W}(Q)-\mathcal{W}(P)=2 i|\mathcal{W}| \sin (\pi / N)$ in our convention, this is exactly the BPS equation for the (isolated) wall, as it should be since the equation for the junction should go into the equation for the wall far away from the "hub."

To begin with, we will calculate the tension $T_{1}$. Since due to the $Z_{N}$ symmetry, the contributions of each of the $N$ sectors in Fig.(11) are identical, it is sufficient to calculate the integral corresponding to one sector,

$$
T_{1} R=2 N \int_{\mathrm{PDHQ}} d \vec{n}_{k} S_{k}
$$

where on $\mathrm{PD}$ and $\mathrm{HQ}$ the integral runs along the circle while on $\mathrm{DH}$ it runs along the straight line. On PD and HQ the superpotential $\mathcal{W}$ assumes the vacuum values

$$
\mathcal{W}=|\mathcal{W}|_{\text {vac }} e^{\pi i / N} \quad \text { on HQ, } \mathcal{W}=|\mathcal{W}|_{\text {vac }} e^{-\pi i / N} \quad \text { on } \mathrm{PD}
$$

and does not change (with exponential accuracy). Therefore,

$$
\int_{\mathrm{PD}+\mathrm{HQ}} d \vec{n}_{k} S_{k}=R|\mathcal{W}|_{\operatorname{vac}}\left\{\int_{-\pi / N}^{-\gamma} \cos \left(\frac{\pi}{N}+\alpha\right)+\int_{\gamma}^{\pi / N} \cos \left(\frac{\pi}{N}-\alpha\right)\right\},
$$

where the angle $\gamma$ is defined in Fig.(四). On the straight line DH we have

$$
\int_{\mathrm{DH}} d \vec{n}_{k} S_{k}=\int_{-L / 2}^{L / 2} d y \operatorname{Re} \mathcal{W}
$$

With the exponential accuracy the fields on the straight line DH satisfy the BPS equations for the isolated wall, see ([9). The solution is known to have an integral of motion (see e.g. [5]), which with our phase convention is nothing but $\operatorname{Re}\{\mathcal{W}\}$. Thus, $\operatorname{Re}\{\mathcal{W}\}$ does not change along the line $\mathrm{DH}$ and reduces to its vacuum value, $|\mathcal{W}|_{\text {vac }} \cos (\pi / N)$, which is one and the same in both vacua, $\mathrm{P}$ and $\mathrm{Q}$. As a result, the sum of two integrals (21) and (22) is

$$
\int_{\mathrm{PDHQ}} d \vec{n}_{k} S_{k}=R|\mathcal{W}|_{\mathrm{vac}} 2 \sin \left(\frac{\pi}{N}-\gamma\right)+L|\mathcal{W}|_{\mathrm{vac}} \cos \frac{\pi}{N}=2 R|\mathcal{W}|_{\mathrm{vac}} \sin \frac{\pi}{N}+O\left(R^{-1}\right)
$$

Moreover,

$$
2|\mathcal{W}|_{\text {vac }} \sin \frac{\pi}{N}=|\mathcal{W}(P)-\mathcal{W}(Q)|=\frac{1}{2} T_{\text {wall }}
$$


where $T_{\text {wall }}$ stands for the tension of the isolated BPS wall. Combining Eqs. (20), (23) and (24) we arrive at

$$
T_{1}=N T_{\text {wall }}
$$

q.e.d.

Now we pass to the discussion of the tension $T_{2}$. According to Eq.(9) for the wall junction tension, $T_{2}$ is given by the integral over the large circle over

$$
T_{2}=-\oint d \vec{x}_{k} a_{k}
$$

where $a_{k}$ is given in Eq. (17) or Eq. (18). Again, we take into account the fact that all $N$ sectors give one and the same contribution, and we will integrate over one sector only, starting from $\mathrm{P}$ and ending at $\mathrm{Q}$. Then

$$
T_{2}=-N \int_{\mathrm{PDHQ}} d \vec{x}_{k} a_{k}
$$

On the intervals $\mathrm{PD}$ and $\mathrm{HQ}$, the fields assume their vacuum values. They do not change (with exponential accuracy), and therefore, $a_{k}=0$, so that there is no contribution to $T_{2}$. A nonvanishing contribution comes from the $\mathrm{DH}$ segment of the integral. On this segment we can disregard the "hub" and other "spokes" of the junction, considering the relevant part of the wall as that of the isolated BPS wall.

In Model I, there is only one field, $X$. In Model II there are $N+1$ fields, $T$ and $M_{\ell}$. However, for the BPS wall, $M_{\ell}$ 's assume real values (see Appendix A) and, thus, the fields $M_{\ell}$ do not contribute. Let us parametrize the fields $X$ and $T$ as $\rho e^{i \eta}$. In both cases $\eta(P)=-\pi / N$ and $\eta(Q)=\pi / N$. Then

$$
T_{2}=-N \int_{\mathrm{DH}} d y \rho^{2}(x, y) \partial_{y} \eta(x, y)=-N\left\langle\rho^{2}\right\rangle(\eta(Q)-\eta(P))=-2 \pi\left\langle\rho^{2}\right\rangle .
$$

Here $\left\langle\rho^{2}\right\rangle$ is the average value of the modulus of the field on the solution on the segment DH, i.e. it is the average over the wall. First, we notice that since $\left\langle\rho^{2}\right\rangle$ is positive, the value of the junction tension $T_{\text {junction }}$ is negative. The only way to escape this, is to have some (complex) fields involved in the junction which rotate in the unnatural direction (i.e. while the "natural" fields in the junction rotate in the anticlock-wise direction, this "exotic" field must rotate clock-wise). Rotation means here winding of the phase, from smaller to larger values. In addition, the "exotic" field must have the average value of the modulus larger than that of the natural fields, so that it can overcome the negative contribution to $T_{\text {junction }}$ coming from the "natural" fields. In all models considered so far of which we are aware, the "exotic" fields do not appear. 2

Second, we note that $\left\langle\rho^{2}\right\rangle$ inside the wall does not differ too much from its vacuum value. For instance, in the Model II (see Appendix A) the vacuum value

\footnotetext{
${ }^{2}$ Let us note in passing that similar consideration determining the sign of the tension $T_{2}$ is applicable to the $U(1)$ vortices of the type considered in [5].
} 
$|T|_{\text {vac }}^{2}=N^{2}$ while in the middle of the wall $|T|_{\text {middle }}=N^{2}[\cos (\pi / N)]^{2}$. The average value $\left\langle\rho^{2}\right\rangle$ lies between these two extremes. At large $N$ the average value tends to its vacuum one, $\left\langle\rho^{2}\right\rangle=N^{2}+O(1)$.

Theorem For BPS junctions $T_{\text {junction }}$ is determined, through the master equation, by the solution for the isolated wall. Unlike $T_{1}$, the domain wall junction is non-holomorphic, and, generally speaking, depends on the details of the wall solution. In the limit $N \rightarrow \infty$ the formula for $T_{\text {junction }}$ becomes universal.

\section{Proof}

The first part of the theorem is proved by the consideration preceding Eq. (28). The last part of the theorem will be established below (see also [11]).

\section{$3 \quad T_{\text {junction }}$ from the isolated wall in Model I}

First, we will illustrate how this works in Model I. Let us consider this model in the limit of large $N$. In this limit the wall junction tension was calculated in [10], $T_{\text {junction }}=-2 \pi N^{2}$. The corresponding calculation is trivial. We are now interested in the leading $1 / N$ correction. It can be found using the solution for the isolated BPS wall obtained in [17]. The field $X$ is parametrized as

$$
X=N\left(1-\frac{\sigma}{N}\right) e^{i \tau / N},
$$

where $\sigma(y)$ is a function of $y$ which falls off from $\sigma_{*}$ in the middle of the wall $(y=0)$ to zero at $y=\infty$. Here $\sigma_{*}$ is the positive root of the equation

$$
\left(1-\sigma_{*}\right) e^{\sigma_{*}}=-1, \quad \sigma_{*} \approx 1.278 .
$$

The phase $\tau$ changes from zero in the middle of the wall $(y=0)$ to $\pi$ at $y=\infty$,

$$
\tau(\sigma)=\pi-\arccos ((1-\sigma) \exp \sigma),
$$

where we modified the solution presented in [17] to bring it in accordance with our convention.

Now in the segment under consideration

$$
a_{y}=(N-2 \sigma) \frac{d \tau}{d y}+O(1 / N),
$$

and

$$
\begin{gathered}
-\int_{\mathrm{DH}} a_{y} d y=-2 \pi N+4 \int_{0}^{\infty} d y \sigma \frac{d \tau}{d y}=-2 \pi N+4 \int_{0}^{\sigma_{*}} \tau(\sigma) d \sigma \\
=-2 \pi N\left(1-\frac{1.77}{N}+O\left(1 / N^{2}\right)\right) .
\end{gathered}
$$


This implies, in turn that

$$
T_{\text {junction }}=-2 \pi N^{2}\left(1-\frac{1.77}{N}+O\left(1 / N^{2}\right)\right) .
$$

The tension in Model I can also be calculated numerically for any value of $N$. In Fig.(5) we compare the numerically determined tension with the leading and next to leading analytic approximations for $N=2^{k}$ and $k=2, \ldots, 10$. The value of $N$ therefore ranges between 4 and 1024. A numerical profile of the domain wall was obtained by simulation of the second order equation of motion for the field $X$ on a discrete lattice, using a forward predicting algorithm. The lattice spacing was taken much smaller than the width of the wall, and at the same time the size of the lattice was chosen much larger than this width. The value of the field was fixed to take on the two vacuum values at the two ends of the one dimensional lattice. A dissipation term was added to the equation of motion so that the field relaxes to the minimum energy configuration. An interpolating profile between the two vacuum expectation values was chosen as the initial condition. Errors due to the finite lattice spacing are well under control because the shape of the wall profile is smooth. Errors due to the finite size of the lattice are suppressed because the wall profile tends to the vacuum expectation values exponentially fast. After the field had come to rest, the junction tension was then obtained from Eq.(27) by numerical integration.

It may be worth a few words to motivate our choice of the second order equation of motion over the first order BPS equation to obtain the numerical domain wall profile. Simulation of the equation of motion yields a domain wall profile even if the wall is not BPS saturated, but in the case of Model I this does not offer an advantage, as the walls are known to be BPS saturated. However, numerical integration of the first order BPS equation is fraught with an instability. This can best be seen from an approximate solution of the BPS equation near a vacuum value of the field. Parametrizing the field as $X=N\left(e^{\pi i / N}+a+b i\right)$, the solution to the linearized BPS equation for $a$ and $b$ (valid for $|a|,|b| \ll 1$ ) is

$$
\begin{aligned}
a & =c_{1} \cos \frac{\pi}{N} e^{-N^{2} y}+c_{2} \cos \frac{\pi}{N} e^{N^{2} y} \\
b & =c_{1}\left(1-\sin \frac{\pi}{N}\right) e^{-N^{2} y}-c_{2}\left(1+\sin \frac{\pi}{N}\right) e^{N^{2} y}
\end{aligned}
$$

where $c_{1}$ and $c_{2}$ are real constants. For a wall connecting the vacuum with $k=1$ at $y=-\infty$ to the vacuum with $k=2$ at $y=\infty, c_{2}=0$. The value of $c_{1}$ is associated with the location of the center of the wall. In simple numerical procedures to integrate the BPS equation, $c_{2}$ will be small, but not equal to zero. As a result, the numerical solution will show runaway behavior far away from the center of the wall. 


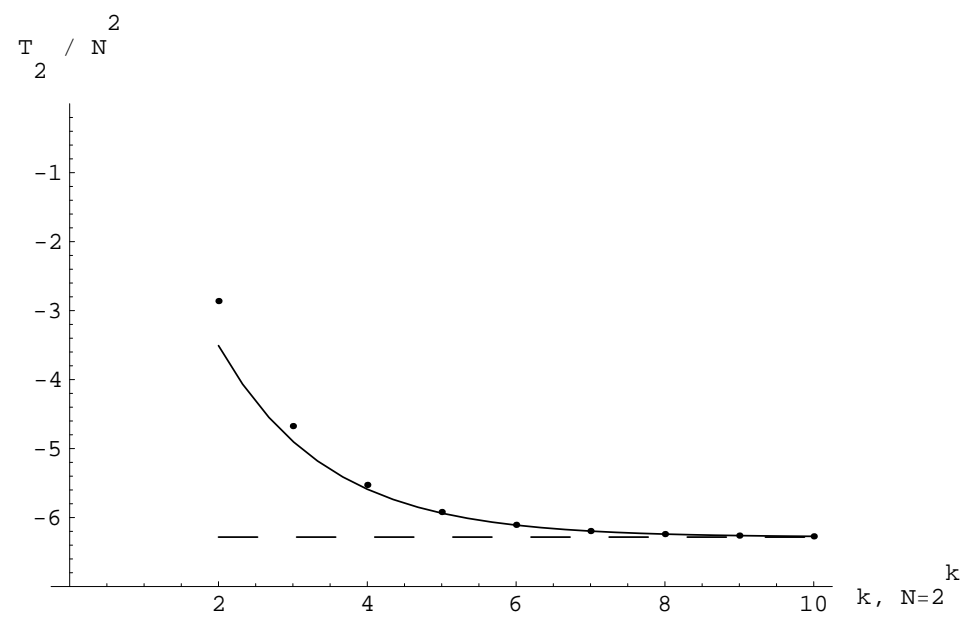

Figure 5: The tension of the junction in Model I as a function of $N$. The dashed line reflects the leading order contribution in $1 / N$. The solid line includes the next to leading order contribution, and the dots represent the numerical calculation.

\section{$4 \quad T_{\text {junction }}$ in Model II}

Although in general $T_{\text {junction }}$ depends on the detailed shape of the domain walls surrounding the junction, this is not the case for Model II. In fact, the tension of the junction in Model II does not depend on $A$ and $B$ and can be calculated exactly even though an analytic solution for the domain wall is not available for generic ratios of $A$ and $B$. The only assumption is that the fields $M_{1}$ and $M_{2}$ are real on the domain wall profile. This is consistent with the explicit domain wall solution for the special ratio of $A$ and $B$ discussed in Appendix $\mathrm{A}$, and it is confirmed by numerical solutions of the domain wall profile for generic ratios. The field $T$ then takes the form $T=-i t+N \cos \pi / N$ on the domain wall, where $t$ is a real function with asymptotic values $t(-\infty)=N \sin \pi / N$ and $t(\infty)=-N \sin \pi / N$. The junction tension is now equal to

$$
T_{2}=-\oint d x_{k} a_{k}=N \int_{-\infty}^{\infty} N \cos \pi / N \frac{d t}{d x} d x=-N^{3} \sin 2 \pi / N
$$

The tension therefore only depends on the asymptotic values of the field $T$ on the domain wall and is independent of the detailed shape of the wall. In this sense, the junction tension $T_{\text {junction }}$ in Model II is similar to the $(1,0)$ tension of domain walls. 


\section{Reshuffling between the $(1,0)$ and $(1 / 2,1 / 2)$ cen- tral charges and the unique expression for $T_{1,2}$}

As was discussed at length in [10], in the case of wall junctions in supersymmetric theories, one deals, in fact, with two central charges - one appears in the anticommutator $\left\{Q_{\alpha} Q_{\beta}\right\}$ (this is the $(1,0)$ central charge), another appears in the anticommutator $\left\{Q_{\alpha} \bar{Q}_{\dot{\beta}}\right\}$ (this is the $(1 / 2,1 / 2)$ central charge). The individual expressions for these central charges are not unique. They depend on the expression for the supercurrent one starts with. This is due to the fact that the effect under discussion is subtle - we are interested in integrals over total derivatives. The supercurrent has an ambiguity. Say, in the Wess-Zumino model with one chiral superfield one can add to the supercurrent the term

$$
\Delta J_{\alpha \beta \dot{\beta}}=-\frac{\sqrt{2}}{3}\left[\partial_{\alpha \dot{\beta}}\left(\psi_{\beta} \phi^{\dagger}\right)+\partial_{\beta \dot{\beta}}\left(\psi_{\alpha} \phi^{\dagger}\right)-3 \varepsilon_{\beta \alpha} \partial_{\dot{\beta}}^{\gamma}\left(\psi_{\gamma} \phi^{\dagger}\right)\right],
$$

which is conserved (nondynamically) and presents a full derivative. It has no impact on the supercharges defined as

$$
Q_{\alpha}=\int d^{3} x J_{\alpha}^{0}, \quad J_{\alpha}^{\mu}=\frac{1}{2}\left(\bar{\sigma}^{\mu}\right)^{\dot{\beta} \beta} J_{\alpha \beta \dot{\beta}}
$$

(for definitions see [5]). The contribution of $\Delta J_{\alpha \beta \dot{\beta}}$ in $Q_{\alpha}$ is of the form $\int d^{3} x \vec{\nabla}(\ldots)$. Adding the term in Eq. (37) one changes both central charges.

If we do not add the term $\Delta J_{\alpha \beta \dot{\beta}}$ in the supercurrent at all (by the way, this will correspond to the canonic energy-momentum tensor), then we get Eq.(9) with $S$ and $a$ defined in Eqs. (16) and (17). Assume that we add to the supercurrent the term $\Delta J_{\alpha \beta \dot{\beta}}$ as in Eq. (37), with the coefficient indicated in this expression. What changes?

It is not difficult to show [5] that the changes are as follows: (ii) the energymomentum tensor appearing in the anticommutator of two supercharges acquires a full-derivative (conserved) term built from the scalar fields; this term is such that $\theta_{\mu}^{\mu}$ now vanishes for purely cubic superpotential, i.e. for the conformally invariant theory; (ii) $\Sigma$ in Eq. (16) now becomes

$$
\Sigma=\mathcal{W}-\frac{1}{3} \Phi \frac{\partial \mathcal{W}}{\partial \Phi}, \quad S_{1,2}=\{\operatorname{Re} \Sigma, \operatorname{Im} \Sigma\}
$$

(iii) simultaneously, the expression $-\oint a_{k} d x_{k}$ in Eq.(9) changes too. Let us keep, for simplicity, the definition of the current (17) intact. Then a "new" master equation takes the form

$$
\frac{M}{\text { length }}=-\frac{1}{3} \oint a_{k} d x_{k}+2 \oint d n_{k} S_{k},
$$

where $S$ is defined in Eq.(39). 
It is instructive to check that, in spite of the reshuffling in the $(1,0)$ and $(1 / 2,1 / 2)$ central charges, the final results for the tensions $T_{1,2}$ remain unambiguous. They are insensitive to this reshuffling. One can do this check explicitly using the analytic solution for the BPS wall which exist in Model II, see Appendix. (A general proof was presented in [10].) As previously, we split the contour integral in $N$ sectors; we will explicitly consider only the transition PQ (Fig.(1)). On the segments PD and $\mathrm{HQ}$ the extra term in the new master equation $\sum \Phi \partial \mathcal{W} / \partial \Phi$ vanishes, since these segments correspond to vacua, where $\partial \mathcal{W} / \partial \Phi=0$. It is only the interval $\mathrm{DH}$ that contributes to the integral over $(-2 / 3) \sum \Phi \partial \mathcal{W} / \partial \Phi$. One can readily convince oneself that the contribution due to $M_{1,2}$ appears only in $\operatorname{Im} \Sigma \equiv S_{2}$, and, thus, drops out. Only the field $T$ is relevant. Due to the fact that only the segments of the type $\mathrm{DH}$ contribute, it is immediately clear that the above extra term in the new master equation changes only the tension $T_{2}$ leaving $T_{1}$ intact.

Now, at $R \rightarrow \infty$ one can replace the DH segment by that for an isolated BPS wall, using explicit expressions collected in Appendix A. In this way we get

$$
-\frac{2}{3} \int d n_{1} \operatorname{Re}\left\{\Phi \frac{\partial \mathcal{W}}{\partial \Phi}\right\}=-\frac{2}{3} N^{2} \sin \frac{2 \pi}{N},
$$

and, consequently,

$$
2 \oint d n_{k} \Delta S_{k}=-\frac{2}{3} N^{3} \sin \frac{2 \pi}{N} \equiv \delta_{2}\left(T_{2}\right)
$$

where

$$
\Delta S_{1,2}=\left\{\operatorname{Re}\left(-\frac{1}{3} \Phi \frac{\partial \mathcal{W}}{\partial \Phi}\right), \operatorname{Im}\left(-\frac{1}{3} \Phi \frac{\partial \mathcal{W}}{\partial \Phi}\right)\right\} .
$$

Using the same approach we readily find that the term

$$
T_{2}=-\oint d x_{k} a_{k}=-N^{3} \sin \frac{2 \pi}{N}
$$

this term was in the old master equation. In the new master equation we have one third of it, i.e. the contribution to $T_{2}$ coming from the axial current is one third of that in Eq. (44),

$$
\delta_{1}\left(T_{2}\right)=-\frac{1}{3} N^{3} \sin \frac{2 \pi}{N} .
$$

Combining this with $\delta_{2}\left(T_{2}\right)$ from Eq. (42), we arrive at

$$
T_{2}=\delta_{1}\left(T_{2}\right)+\delta_{2}\left(T_{2}\right)=-N^{3} \sin \frac{2 \pi}{N},
$$

exactly the same result for $T_{\text {junction }}$ that was obtained from the old master equation where only the axial current contributed to it. The reshuffling in the central charges does take place; there is no impact on the physical quantities, however. 


\section{BPS saturation of junctions in Models I and II}

In this section we consider the question whether the domain wall junctions in Models I and II saturate the BPS bound. For certain limiting values of the parameters, the BPS equations for domain wall junctions in Models I and II allow an analytic solution. This is the case for the $N \rightarrow \infty$ limit in model I, as was shown in [10]. And in Appendix B we present an analytic solution for the triple junction in Model II, which can be obtained when $N$ is a multiple of 3 and for a particular ratio of $A$ and $B$. This junction is a generalization of the analytic solution in [13]. For $N=3$ the triple junction is also the basic junction. The above values of the parameters seem only special because they allow an analytical solution, and not for any other reason. Moreover, for any value of the parameters there are sensible approximate solutions to the BPS equation far away from the center of the junction (domain walls surrounding the center) and near the center (string-like solutions). All this information strongly suggests that the junctions are BPS saturated for all values of the parameters.

In order to further test this hypothesis, we performed a numerical analysis. We simulated the second order equations of motion for both Models I and II on a lattice using a simple forward predicting algorithm. The procedure is a two-dimensional generalization of the procedure described in Sect.(3) to generate numerical domain wall profiles. The lattice spacing was chosen to be much smaller than the characteristic size $l$ of the junctions, and at the same time the size of the lattice was much larger than $l$. We put the appropriate domain wall profiles (numerically generated) on the edges of the lattice as boundary conditions. A dissipation term was added to the equations of motion, so that the field configuration relaxes to the configuration with lowest energy consistent with the boundary conditions.

We then determined whether the numerically obtained junction profile saturated the BPS bound by comparing the energy of the configuration to $E(R)=T_{1} R+T_{2}$. For Model I, $T_{1}=4 N^{4} /(N+1) \sin \pi / N$, and $T_{2}$ was determined numerically as in Sect.(3). For Model II, $T_{1}=4 N^{3} B \sin \pi / N$ and $T_{2}$ is given in Eq.(36). Numerical errors due to the finite lattice spacing were well under control because the junction shape is smooth, and errors due to the finite size of the lattice were suppressed because the junction profile tends toward the domain wall solutions exponentially fast away from its center.

We performed this procedure for Model I with $N=4$, and for Model II with $N=4$ and various values of the parameters $A$ and $B$. In each case we found that the junction was indeed BPS saturated. This fact, in conjunction with the analytical results, lends strong support to the conjecture that the basic junctions we have discussed in Models I and II are BPS saturated for all values of the parameters. 


\section{Comments on Stability of the BPS wall junc- tions}

A general question which deserves a brief discussion is the stability (local and global) of the BPS wall junctions. In this issue two features are crucial: (i) the two-dimensional manifold perpendicular to the junction axis (the plane) is noncompact, i.e. the junction "spokes" extend to infinity; (ii) the wall junction is not a two-dimensional soliton; rather, each "spoke" represents a wall which extends in the direction perpendicular to the plane. This extension is infinite.

As was discussed above, the mass of the BPS wall junction can be expressed in terms of two central charges which reduce, in turn, to the contour integral over the large circle (see Fig.(66.a), where we consider a $Z_{6}$ configuration, for definiteness). This means that any localized perturbation of the junction configuration, both in the plane (Fig.6.6), and in the perpendicular direction, will lead to an encreased energy, i.e. the BPS wall junction is stable against such localized perturbations.

A global perturbation, when two "spokes" approach each other and then eventually fuse into one, is energetically favorable (Figs. (6.c) and (6.). This is due to the fact that the isolated BPS wall tension for the transition from the vacuum I to the vacuum $\mathrm{J}$ has the form

$$
T_{\mathrm{IJ}}=2\left|\mathcal{W}_{\mathrm{J}}-\mathcal{W}_{\mathrm{I}}\right|=2\left|\mathcal{W}_{\text {vac }}\right| \times\left|e^{2 \pi i J / N}-e^{2 \pi i I / N}\right|,
$$

in the models under consideration, and, hence

$$
T_{\mathrm{I}-\mathrm{II}}+T_{\mathrm{II}-\mathrm{III}}>T_{\mathrm{I}-\mathrm{III}} .
$$

In other words, eating up an intermediate vacuum we lower the energy of the configuration.

Were the wall sizes finite, the configuration with six spokes in Fig.(6.a) could quantum-mechanically tunnel into the configuration with five spokes (Fig.(6.).d), under the barrier. However, since the walls are infinite in both dimensions, this cannot happen. The boundary conditions at infinities make the BPS wall junctions stable both classically and quantum-mechanically. For further consideration see Ref. [14].

\section{Conclusions}

In supersymmetric models with the spontaneously broken $Z_{N}$ symmetry we developed techniques for (i) establishing the BPS nature of the wall junctions of the "hub and spokes" type and for (ii) calculating the tensions $T_{1}$ and $T_{\text {junction. }}$. It is shown that in "natural" models $T_{\text {junction }}<0$. The conditions under which the "unnatural" sign $T_{\text {junction }}>0$ can occur are discussed. 

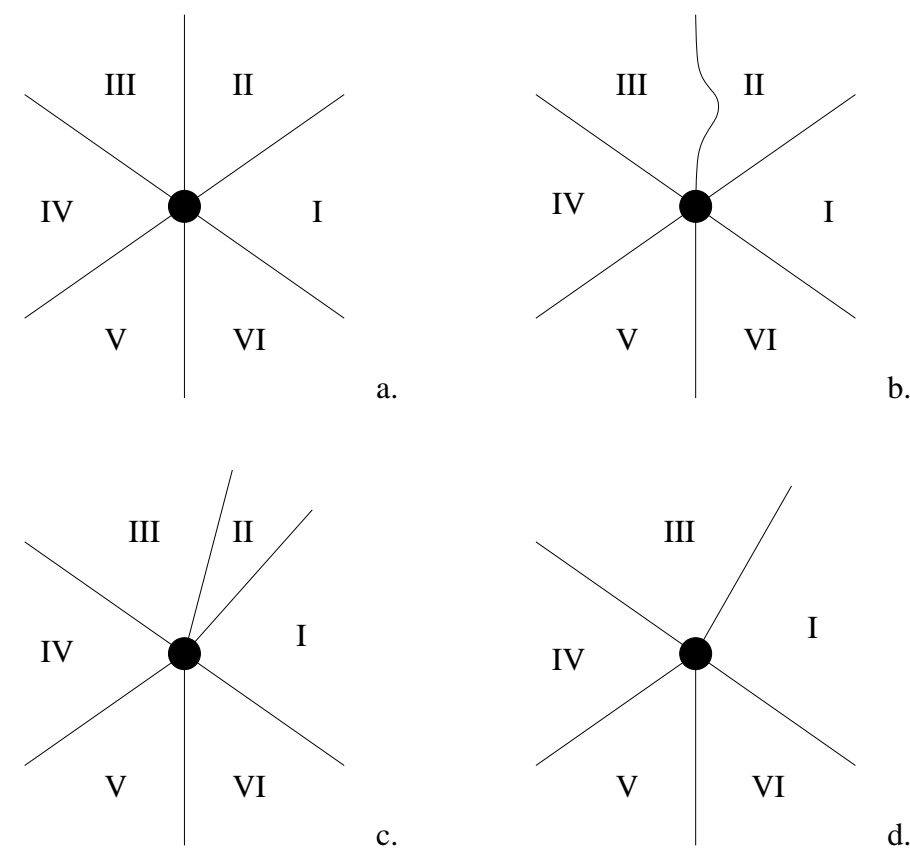

Figure 6: Stability of the wall junction. a.) Regular BPS junction in a model with $Z_{6}$. b.) A localized deformation of the wall junction. c.) Squeezing out the vacuum II. d.) The wall junction with the vacuum II missing.

\section{Acknowledgements}

One of the authors (M.S.) acknowledges useful discussions with Zurab Kakushadze which took place at ITP, Santa Barbara, in the course of the program "Supersymmetric Gauge Dynamics and String Theory". This work was supported in part by the Department of Energy under Grant No. DE-FG02-94ER40823 and by National Science Foundation under Grant No. PHY94-07194.

\section{Appendix A}

Here we present an analytic solution for the BPS wall in Model II which can be readily found for a specific ratio of the coefficients $A$ and $B$, namely

$$
A=\frac{B}{[2 N \sin (\pi / N)]^{2}} .
$$

For what follows it is convenient to introduce the parameter $\lambda$ which determines the width of the wall,

$$
\lambda=l^{-1}=\frac{B}{2 \sin (\pi / N)} .
$$

Note that at large $N$ the parameter $\lambda$ scales as $N$; correspondingly, $l \sim N^{-1}$. 
We will consider the wall interpolating between the vacuum 1 ("initial" vacuum) at $y=-\infty$ and the vacuum 2 ("final" vacuum) at $y=+\infty$.

The vacuum 1 lies at

$$
T=N e^{-\pi i / N}, \quad M_{1}=\sqrt{\frac{B}{A}}, \quad M_{2}=M_{3}=\ldots=0 .
$$

The vacuum 2 lies at

$$
T=N e^{\pi i / N}, \quad M_{2}=\sqrt{\frac{B}{A}}, \quad M_{1}=M_{3}=\ldots=0 .
$$

For the superpotential in the vacua 1 and 2 we have

$$
\mathcal{W}(1)=B N^{2} e^{-\pi i / N}, \quad \mathcal{W}(2)=B N^{2} e^{\pi i / N},
$$

so that

$$
\Delta \mathcal{W}=2 i B N^{2} \sin (\pi / N) .
$$

Correspondingly, the BPS equations for the fields $T, M_{1,2}$ have the form

$$
\dot{T}=i \frac{\partial \overline{\mathcal{W}}}{\partial \bar{T}}, \quad \dot{M}_{\ell}=i \frac{\partial \overline{\mathcal{W}}}{\partial \bar{M}_{\ell}} .
$$

Here the dot denotes differentiation over $y$. The solution of these equations is

$$
T=N \cos \frac{\pi}{N}+i N\left(\sin \frac{\pi}{N}\right) \tanh (\lambda y)
$$

and

$$
M_{1}=\sqrt{\frac{B}{A}} \frac{1-\tanh (\lambda y)}{2}, \quad M_{2}=\sqrt{\frac{B}{A}} \frac{1+\tanh (\lambda y)}{2} .
$$

\section{Appendix B}

Here we present an analytic solution for BPS walls and triple junctions in Model II, with $N$ a multiple of 3 . An analytic solution for the triple junction exists if the coefficients $A$ and $B$ satisfy the relation

$$
A=\frac{B}{3 N^{2}}
$$

Note that the ratio of $A$ and $B$ depends on $N$, in contrast with what was assumed in the main text. For this particular ratio of $A$ and $B$, the parameter $\lambda$ which determines the width of the wall and the size of the junction takes the form

$$
\lambda=l^{-1}=\frac{B}{\sqrt{3}} .
$$


We will consider a junction that interpolates between three of the $N$ vacua. Vacuum 1 lies at

$$
T=N e^{-\pi i / N}, \quad M_{1}=\sqrt{\frac{B}{A}}, \quad M_{k}=0, k \neq 1,
$$

vacuum 2 lies at

$$
T=N e^{2 \pi i / 3} e^{-\pi i / N}, \quad M_{N / 3+1}=\sqrt{\frac{B}{A}}, \quad M_{k}=0, k \neq N / 3+1,
$$

and vacuum 3 lies at

$$
T=N e^{4 \pi i / 3} e^{-\pi i / N}, \quad M_{2 N / 3+1}=\sqrt{\frac{B}{A}}, \quad M_{k}=0, k \neq 2 N / 3+1 .
$$

Let us first focus on a domain wall interpolating between vacua 1 and 2 along the $\hat{y}$ axis. Such a wall is a solution to the BPS equations

$$
\dot{T}=i e^{i \delta} \frac{\partial \overline{\mathcal{W}}}{\partial \bar{T}}, \quad \dot{M}_{1}=i e^{i \delta} \frac{\partial \overline{\mathcal{W}}}{\partial \bar{M}_{1}}, \quad \dot{M}_{N / 3+1}=i e^{i \delta} \frac{\partial \overline{\mathcal{W}}}{\partial \bar{M}_{N / 3+1}},
$$

where the dot indicates differentiation with respect to $y$. When the phase takes the value $\delta=\pi / 3-\pi / N$, the wall configuration tends to vacuum 1 at $y \rightarrow-\infty$ and to vacuum 2 at $y \rightarrow \infty$. The analytic solution to the BPS equation is

$$
T=\frac{N}{2} e^{-\pi i / N}\left(e^{\pi i / 3}-\sqrt{3} e^{-\pi i / 6} \tanh (\lambda y)\right)
$$

and

$$
M_{1}=\sqrt{\frac{B}{A}} \frac{1-\tanh (\lambda y)}{2}, \quad M_{N / 3+1}=\sqrt{\frac{B}{A}} \frac{1+\tanh (\lambda y)}{2} .
$$

Having the solution for the wall in hand, we now continue with the triple junction. In accordance with the convention in the main text, our choice of $\delta$ implies that the spoke in between the first and the second vacuum points in the direction of the positive $\hat{x}$ axis. For $N=3$, the phase $\delta=0$, as in the main text. For other values of $N$, insisting on $\delta=0$ would lead to a very awkward orientation of the junction. The junction is the solution to the BPS equations

$$
2 \frac{\partial T}{\partial \zeta}=e^{i \delta} \frac{\partial \overline{\mathcal{W}}}{\partial \bar{T}}
$$

and

$$
2 \frac{\partial M_{1}}{\partial \zeta}=e^{i \delta} \frac{\partial \overline{\mathcal{W}}}{\partial \bar{M}_{1}}, \quad 2 \frac{\partial M_{N / 3+1}}{\partial \zeta}=e^{i \delta} \frac{\partial \overline{\mathcal{W}}}{\partial \bar{M}_{N / 3+1}}, \quad 2 \frac{\partial M_{2 N / 3+1}}{\partial \zeta}=e^{i \delta} \frac{\partial \overline{\mathcal{W}}}{\partial \bar{M}_{2 N / 3+1}}
$$


with $\zeta=x+i y$. Defining

$$
g(x, y)=e^{-\lambda(y-x / \sqrt{3})}+e^{\lambda(y+x / \sqrt{3})}+e^{-2 \lambda x / \sqrt{3}},
$$

the triple junction BPS configuration takes the form

$$
T=N e^{-\pi i / N} \frac{e^{-\lambda(y-x / \sqrt{3})}+e^{2 \pi i / 3} e^{\lambda(y+x / \sqrt{3})}+e^{4 \pi i / 3} e^{-2 \lambda x / \sqrt{3}}}{g(x, y)},
$$

and

$$
M_{1}=\sqrt{\frac{B}{A}} \frac{e^{-\lambda(y-x / \sqrt{3})}}{g(x, y)}, \quad M_{N / 3+1}=\sqrt{\frac{B}{A}} \frac{e^{\lambda(y+x / \sqrt{3})}}{g(x, y)}, \quad M_{2 N / 3+1}=\sqrt{\frac{B}{A}} \frac{e^{-2 \lambda x / \sqrt{3}}}{g(x, y)} .
$$

For $x \rightarrow \infty$ the solution tends to the domain wall configuration along the $\hat{y}$ axis

which was discussed before. For $x \rightarrow-\infty$ and $y=0$ the field configuration tends towards vacuum 3 . The tension for the triple junction is

$$
T_{2}=-\frac{3}{2} \sqrt{3} N^{2}
$$

This is an exact result, valid for any value of $A$ and $B$, not just the special ratio for which the analytic solution for the junction is obtained. For $N=3$ this result agrees with the tension presented in Eq.46) in the main text.

\section{References}

[1] G. Dvali and M. Shifman, Phys. Lett. B396, 64 (1997). Erratum-ibid B407, 452 (1997).

[2] E. Witten, Phys. Rev. Lett. 81, 2862 (1998).

[3] M. Shifman, Phys. Rev D 59, 021501 (1998).

[4] E.R.C. Abraham and P.K. Townsend, Nucl. Phys. B351, 313 (1991).

[5] B. Chibisov and M. Shifman, Phys. Rev. D56, 7990 (1997); Erratum-ibid. D58, 109901 (1998).

[6] G. W. Gibbons and P. K. Townsend, Phys. Rev. Lett. 83, 1727 (1999).

[7] S. M. Carroll, S. Hellerman and M. Trodden, Domain wall junction are 1/4BPS states, hep-th/9905217.

[8] J.A. de Azcárraga, J.P. Gauntlett, J.M. Izquierdo and P.K. Townsend, Phys. Rev. Lett. 63, 2443 (1989). 
[9] S. Ferrara and M. Porrati, Phys. Lett. B423, 255 (1998).

[10] A. Gorsky and M. Shifman, More on tensorial central charges in $N=1$ supersymmetric theories (BPS wall junctions and strings), hep-th/9909015.

[11] G. Gabadadze, M. Shifman, D-Walls and junctions in Supersymmetric Gluodynamics in the large $N$ limit suggest the existence of Heavy Hadrons, hepth/9910050.

[12] D. Binosi, Domain wall junctions from a distance, hep-th/9910057.

[13] H. Oda, K. Ito, M. Naganuma and N. Sakai, An exact solution of BPS domain wall junction, hep-th/9910095.

[14] D. Binosi and T. ter Veldhuis, Domain wall junctions in a generalized WessZumino model, hep-th/9912081.

[15] G. Veneziano, S. Yankielowicz, Phys. Lett. 113B, 231 (1982).

[16] G. Dvali, G. Gabadadze and Z. Kakushadze, BPS domain walls in large $N$ supersymmetric QCD, hep-th/9901032.

[17] G. Dvali and Z. Kakushadze, Nucl. Phys. B537, 297 (1999).

[18] P. Fendley, S. Mathur and C. Vafa, Phys. Lett. B243, 257 (1990).

[19] A.V. Smilga and A.I. Veselov, Phys. Lett. B428, 303 (1998).

[20] P.M. Saffin, Tiling with almost-BPS junctions, hep-th/9907066.

[21] D. Bazeia and F.A. Brito, Bags, junctions, and networks of BPS and non-BPS defects, hep-th/9912015. 